\title{
Exploring learner variability: experiences of students with cognitive disabilities in post-secondary STEM
}

\author{
Westley James ${ }^{1}$, Kamryn Lamons ${ }^{1}$, Jillian Schreffler ${ }^{2}$, Eleazar Vasquez III $^{2}$ and Jacquelyn J. Chini ${ }^{1}$ \\ ${ }^{1}$ Physics Department, University of Central Florida, 4000 Central Florida Blvd, Orlando, FL, 32816 \\ ${ }^{2}$ Toni Jennings Exceptional Education Institute, University of Central Florida, 4000 Central Florida Blvd, Orlando, FL, \\ 32816
}

\begin{abstract}
Students with disabilities make up approximately $10 \%$ of post-secondary students pursuing STEM degrees. Unfortunately, little is known about these students' experiences in post-secondary STEM classes. We are starting to address this knowledge gap by investigating the barriers, supports, and successes students with cognitive disabilities experience in college physics and chemistry courses. We interviewed both students with ADHD and STEM instructors about student experiences in introductory physics and chemistry courses. Initial findings suggest such courses pose a significant challenge for these students compared to their other courses and that certain instructor/student practices can reduce this challenge. Additionally, instructors report that while they may be unable to identify students with cognitive disabilities in their classrooms, they find that the difficulties these students encounter are found in varying degrees across all students. Thus, practices that benefit students with disabilities are likely to benefit all students.
\end{abstract}

\section{INTRODUCTION}

Students with disabilities (SWD) are an understudied population in post-secondary STEM, possibly due to the unseen nature of cognitive disabilities that make up the majority of this population [1,2]. However, SWD form a sizable portion of the college population. As of 2011, SWD made up $11 \%$ of the post-secondary student population overall and $10 \%$ of STEM majors [2,3]. Attention Deficit/Hyperactive Disorder (ADHD) is an increasingly common diagnosis that is identified by a cognitive deficit in the ability for long term planning, focusing on tasks, and organization [4]. Across the United States, $18 \%$ of postsecondary SWD have been diagnosed with ADHD [2]. At the University of Central Florida (UCF), ADHD diagnoses make up $48 \%$ of those with cognitive disabilities enrolled in Student Accessibility Services (SAS), our disability services office. Previous studies have found that these students face difficulties, such as additional time required to complete assignments and exams and perceptions of working harder to achieve good grades [5]. How these and other challenges associated with ADHD manifest in STEM learning has not been well investigated [6,7].

In this study, we seek to understand the STEM learning environment from the perspective of students with ADHD to inform instructional practices. Consistent with standpoint theory, we start by interviewing students to improve our understanding of the specific barriers and successes students with ADHD encounter in learning post-secondary STEM [8]. These interviews revealed the critical importance of testing accommodations for this group. Additionally, while the students did not feel excluded or unwelcome in their interactions with peers and instructors, using accommodations caused feelings of discomfort about possibly benefiting from an unfair advantage for some.
We also interviewed STEM faculty and graduate teaching assistants (TAs) to explore their experiences with SWDs and perspectives on accommodations. We found similar results to previous studies in post-secondary education, such as instructors recognizing student diversity in their classrooms, while also feeling untrained for how to support SWD and therefore unable to best help them $[7,9,10]$.

A brief note on language: we have chosen "people-first" language ("students with disabilities") as this is the trend in the exceptional education community. When referring to specific students, we will adopt the language preferred by that individual.

\section{METHODS}

We recruited students with cognitive disabilities who were enrolled in introductory physics or chemistry courses at UCF. Initial recruitment ran through SAS, but we later cast a wider net by recruiting from the entire courses and asking students to self-check that they fit the eligibility guidelines. We interviewed students at both the beginning and end of the semester and gave interviewees a small remuneration.

We recruited four student participants, all of whom selfreported as having ADHD. Table 1 displays participants' pseudonyms and the physics/chemistry course they were taking that semester.

TABLE 1. Student Participant Information

\begin{tabular}{cc}
\hline \hline Pseudonym & Current STEM class \\
\hline Annie & Algebra Based Physics 2 (SCALE-UP) \\
Beth & Algebra Based Physics 2 (SCALE-UP) \\
Cassy & Chemistry 2 Traditional Lecture \\
Dan & Calculus Based Physics 2 (traditional) \\
\hline \hline
\end{tabular}


We recruited four instructors from transformed courses in the introductory physics and chemistry sequences at UCF. Specifically, we recruited two SCALE-UP physics faculty instructors and two inquiry-based chemistry laboratory TAs. Instructors were recruited into a broader project and agreed to participate in training and engage in course improvements to make learning more accessible for SWDs. These instructors were interviewed to provide a baseline understanding of the instructors' experiences with SWDs.

Based on previous literature [6,7,9-12] and our own research interests, we created two, one-hour, semi-structured interview protocols, one for SWDs and the other for instructors. These both targeted four specific areas: 1) self and faculty supports and successes for learning, 2) student barriers to learning, 3) opinions/experiences of accommodations, and 4) the culture of acceptance towards SWD by other students and faculty. All interviews were conducted in person by the first author.

Interviews were transcribed and analyzed for emergent themes. Two researchers independently identified significant themes and then compared findings to reach agreement. This process of analysis increases the reliability of interviewee response interpretation and is corroborated by its extensive use in previous studies of this type [7,12].

The themes reported in this paper were found to be remarkably consistent across participants, with at least two participants mentioning them explicitly and at least one other implicitly. Most findings were also consistent with previous literature. However, it is unique that no students reported resistance about using accommodations [5].

\section{SUPPORTS, SUCCESSES, AND BARRIERS}

Instructors expressed an inability to specifically identify students with cognitive disorders in their classes, however they also reported that many students had difficulty in attention to task and problem solving, key difficulties found in students with cognitive disabilities [4]. Thus, while describing supports/successes and barriers for learning, we focus on the student interviews.

Four main categories emerged from our analysis of the student interviews: 1) learning in class, 2) learning out of class, 3) motivation, and 4) expression of understanding.

\section{A. Learning in class}

Lecture was the critical barrier for these students because it requires extended periods of focus. As Annie stated, “...it's really easy for me to get distracted, so when someone's lecturing,... the moment I like lose focus for a little bit and I come back and I don't know what's going on." This difficulty, combined with the frequent use of lecture in these STEM classes, resulted in students doing most of their learning outside of class. Long sessions of instructor-centered problem-solving resulted in similar difficulties for Beth: "we have like recitation problems and the problem gets, starts getting kind of long... and so those times, my attention span would just kinda go... you start to get like fatigued." Beth later described how these difficulties can be uniquely challenging in STEM: "And then at the end, he'll put in the number. But if you don't, if you get lost at one of the steps with the letters then you're screwed for the rest." These concerns match with the known difficulties of attention to task for students with ADHD [4].

Teacher practices that helped the students in class were strategies that helped to break up the monotony of lecture, such as clicker questions or student-centered problemsolving. A "good lecturer" also assisted with focus, with attributes including: being empathetic, admitting mistakes, making content material relevant, and giving opportunities to interact with the material.

\section{B. Learning out of class}

Time was an overarching, acute concern as these students reporting taking much longer to study the material than the average students. Annie shared, "Cause generally what takes another person an hour to study, it'll take me like 2-4 hours to study...it takes me a while because I just get distracted." Annie reported this to be of specific concern in physics: "if it's something like physics where it's not just memorization, you have to go through the problems I feel like you don't have enough time to do that kind of stuff...but for biomedical sciences I feel like a lot of those classes are just memorization."

By doing most of their learning out of class, the students can go at their own pace and plan out their learning, helping to address the time concern. Every student interviewed heavily relied on a planner to schedule their studying and class work. Dan described the critical nature of this strategy when he said, "... [the planner] was something that I, if I hadn't started doing, my life would, I don't know where it'd be." However, to plan well the students needed to know when assignments would be due and when evaluations would happen. Hence, it is important that instructors have an organized syllabus with all these dates listed for easy access. Dan explained "but to know all that information at the beginning would be perfect because if you knew... there's going to be an assignment due for this chapter, you know, okay, I can plan out everything following up to that."

Interestingly, each student preferred a different mode of study: textbook, teacher notes, YouTube videos, etc. Regardless of the format, the students had to develop study strategies to best interact with the material, and these were often self-taught. For Dan and Beth, this was of special concern in STEM as they expressed a lack of training and intuition in how to think about the material. As Dan said, "... At first when I would read the chapters it was, it was hard because um some of the concepts are ... they're not intuitive, you can't really see them happen and that was the biggest challenge at first." The result of this is more time lost, as the students now must not only learn the material on their own, but also discover how to learn the material. Beth proposed a 
solution to this: "having a foundational week would be the most amazing thing, I think, not just for people with focus issues, but students in general... if the teacher is telling you the first week, 'Hey! We're not going to jump into the material right now, but I really want you to understand how this class is set up, how you can be successful in this class." By spending time preparing students to interact with the material, the instructor helps students with ADHD, while supporting all students for success in their class.

\section{Motivation}

The students reported well known practices for increasing motivation, such as making the information relevant to their interests or the instructor showing passion for the material. However, there was an interaction between the student's disability and motivation in the areas of focus and time to complete tasks. Regarding focus, Annie reported a difficulty focusing due to the instructor speaking softly and holding long lectures. She expressed her frustration, “...I want to learn but it's just going so shittily I don't want, I don't care anymore". For Beth, she reported difficulty with staying on pace with the course because she required additional time to process this material. She explained that this impacted her motivation: "And I would easily get discouraged about [unintelligible] at the pace of the class. I would be like, I'm depressed, I'm sad, I'm not going to study because I'm not going to get it." Many students reported that accepting their disability as part of who they are, rather than a weakness, helped them to overcome these barriers. For example, Dan explained "...I've realized that, you know, this weakness has become my strength." Since difficulties in focus and time to complete tasks were also identified to be learning barriers, by addressing these an instructor can both help to prevent demotivation and increase accessibility to learning for students with ADHD.

\section{Expression of Understanding}

Tests were of significant concern for these students in STEM courses, specifically in their capability to complete them in the allotted time. Their preparation work would give them the content understanding, however their difficulties in focusing often resulted in running out of time during the test. Annie expressed how this problem existed for her when she stopped using the extra test time accommodation: "I couldn't finish the material even though I really knew what was going on but um and it was kind of like, it was very annoying because I could've done so much." The students also reported concerns about the time needed to perform mathematical calculations. Dan explained, "I am not quick with math. I can learn it, and I can do a hell of a lot of practice problems, but at the same time I really, I need to take my time when I'm doing these problems."

All the participants expressed that this difficulty appears to be well addressed through the accommodations of extra test time and low distraction test environment provided by
SAS. However, several participants simultaneously reported feelings of unfairness in using this accommodation. Annie described: "... in this past semester I felt, I felt really bad about using SAS because [unintelligible] I do have ADHD ... but it's not fair like I don't like looking at my peers and being like I'm doing better because I have whatever." This leaves us with the concern that while we are addressing these student's needs through accommodations, we simultaneously may be supporting feelings of disparity and inequality in ability.

\section{ACCOMMODATIONS}

The accommodations used by these students included extra test time, a low distraction test environment, note taking services, and audio recording the class. The testing accommodations were both the most used and the most effective in application.

Extra test time and the low distraction test environment were reported to be beneficial to the point of being necessary for their success in the course. Dan explained, "honestly if it wasn't for this class, for these accommodations, I think I'd have to undergo another withdraw from one of the classes." By removing the stress of time, the students were able to focus on expressing that they understood the material.

Students reported that the accommodations were easy to make use of, as they just had to report the need online and SAS handled the details. Instructors only interacted with the testing accommodations, and they reported an ease of use in providing them: "And the awesome thing about our SAS office is that they take care of all of that. Right, so if a student needs extra time or quiet space or something to be working they get all of that, and it's no problem, and it's all done at their office, and they administer the tests and get them back to me."

Each student participant reported never encountering a teacher who was unwilling or resistant to provide accommodations. As Annie said, “...when they go through the syllabus, they always make sure to have a part about like uh when you need accommodations ... please uh email me or please, you know, come up to talk to me to make sure we can figure things out." Likewise, every instructor reported a willingness in their department to provide accommodations with statements such as: "All of faculty that I'm aware of are happy to go along with the accommodations when they're asked for." These results are surprising in light of the previous literature which reports SWDs encountering barriers to instructors giving accommodations $[9,10,13]$. This may be unique to UCF and due to the ease of use of SAS, as reported by both students and instructors. It is also possible that only students who had positive experiences volunteered for interviews.

\section{CULTURE}

All of our student participants reported that they never felt outwardly unwelcome or alienated by students or faculty 
at UCF. However, Dan and Beth both mentioned selfimposed feelings of being unwelcome. Dan expressed, "but it's not that people say anything or do anything that make me feel unwelcome, I just make myself feel unwelcome." These feelings stemmed from their reported feelings of deficiency compared to other students and could be alleviated by "owning" their disability. For example, Annie did not report these feelings and comments, but rather stated: "I don't care that I have a disability, a disability because it just it has its own, it has its own things that are really good about it."

All of the students recommended that instructors have a syllabus section for students with disabilities and accommodations. This practice helped improve selfperception and gives awareness of SAS. Cassy described, "on the first day of class, every single one of my professors was like if you ha-need access...need the time, like I'm fine with like the [accommodations] go do it... It makes me feel better about myself."

A practice the instructors found to be helpful was to recommend SAS services to students who may need them. The services SAS provides are communicated at student orientation, however instructors still encountered students who would benefit from the accommodations but were not using them. A significant note is that the instructors all reported the students being welcome to this information, as opposed to being alienated or offended. As one professor reported, "I generally have not had issues with students feeling bad about it. They, you know, they have generally uh been happy to um go get the help that they need."

\section{DISCUSSION AND IMPLICATIONS}

Time was the most critical factor for these students with ADHD due to their difficulty with focusing on one task for an extended time. The students reported that this is further compounded in STEM due to the increased difficulty of the material and the requirement for understanding versus memorization. By giving organized and accessible material, instructors can help reduce the time investment needed to study the material. It is also recommended that instructors give guidance to students on how to approach and interact with the course material for learning. Planning studying and class work was a necessary strategy for our participants, and instructors can facilitate this by providing a schedule for the class material and assessments. To make in class learning more accessible, instructors should be mindful of time spent talking at students. Breaks or activities interspersed in lecture can reduce the difficulty to focus in class for all students.

Accommodations for tests such as extra test time and a low distraction environment are needed; however, they may cause feelings of inequality or guilt in some students. In this study, instructors were comfortable providing accommodations and talking to students about them. Our recommendations are to give outside of class assignments, thereby allowing all students flexibility in how much time to spend on the assignment, and to encourage feelings of equality in ability. This latter point can be achieved by including a section in the syllabus, or making statements in class, that support and acknowledge the use of accommodations.

Every student reported that they never felt outwardly unwelcome in any class by either students or instructors. This is an encouraging result for our classroom environment at UCF. However, the fact that two students reported selfimposed feelings of disparagement raises the concern that a cultural stigma for having a disability still exists.

Future works should aim to corroborate the findings presented in this study, along with seeking to understand the experiences of students with disabilities other than ADHD. To build on this, studies should seek to evaluate the effectiveness of training faculty in implementing practices that increase the accessibility to learning and encourage a welcoming classroom environment. Limitations to this study include the small sample and single institution; future work should include more students at a variety of institutions.

\section{ACKNOWLEDGEMENTS}

This work is supported by NSF DUE No. 1612009.
[1] K. D. Roberts, H. J. Park, S. Brown, and B. Cook, J. Postsecond. Educ. Disabil. 24, 5 (2011).

[2] T. D. Snyder, C. de Brey, and S. A. Dillow, Natl. Cent. Educ. Stat. (2016).

[3] K. Raue and L. Lewis, Natl. Cent. Educ. Stat. (2011).

[4] R. A. Barkley, Attention-Deficit Hyperactivity Disorder: A Handbook for Diagnosis and Treatment (Guilford Publications, 2014).

[5] L. J. Lewandowski, B. J. Lovett, R. S. Codding, and M. Gordon, J. Atten. Disord. 12, 156 (2008).

[6] J. M. McGuire and S. S. Scott, J. Postsecond. Educ. Disabil. 19, 124 (2006).

[7] R. D. Black, L. A. Weinberg, and M. G. Brodwin, Except. Educ. Int. 25, 1 (2015).
[8] L. J. Davis, The Disability Studies Reader (Psychology Press, 1997).

[9] L. Cook, P. D. Rumrill, and M. Tankersley, Int. J. Teach. Learn. High. Educ. 21, 84 (2009).

[10] P. W. Dowrick, J. Anderson, K. Heyer, and J. Acosta, J. Vocat. Rehabil. 22, 41 (2005).

[11] M. V. Izzo, A. Murray, and J. Novak, J. Postsecond. Educ. Disabil. 21, 60 (2008).

[12] J. M. McGuire and S. S. Scott, Learn. Disabil.Multidiscip. J.- 14, 21 (2006).

[13] S. Rao and B. C. Gartin, J. Vocat. Spec. Needs Educ. 25, 47 (2003). 